\title{
Cytokine Analysis of Eosinophilic Ascites in Systemic Lupus Erythematosus
}

\author{
Mamoru Honda $^{1}$ D $\cdot$ Takako Asano $^{1} \cdot$ Daiji Takajo $^{1} \cdot$ Kosei Takada $^{1} \cdot$ Mari Nakamura $^{1} \cdot$ Kanako Sekinaka-Mitsui $^{1} \cdot$ \\ Hajime Wakamatsu $^{1}$. Shigeaki Nonoyama ${ }^{1}$
}

Received: 27 May 2019 / Accepted: 25 September 2019/Published online: 11 November 2019

(C) Dr. K C Chaudhuri Foundation 2019

To the Editor: A 13-y-old girl developed recurrent abdominal pain, diarrhea and massive ascites at disease onset. Abdominal computed tomography and ultrasonography showed ascites and edematous intestine of serosal side. Abdominal puncture revealed eosinophilic ascites (eosinophils $312 / \mu \mathrm{L}$ ). Although eosinophilic gastroenteritis (EGE) was the most suspected diagnosis, systemic lupus erythematosus (SLE) was also suspected because mild leukopenia, proteinuria, and hypocomplementemia were noted intermittently (Supplementary Fig. 1). Anti-nuclear antibody (speckled type) was elevated (1:320), whereas anti-dsDNA and anti-Sm antibodies were within the normal range. Further, she presented with oral ulcer and fulfilled the American College of Rheumatology 1997 Revised Classification Criteria for SLE [1]. Prednisolone (PSL) $(1 \mathrm{mg} / \mathrm{kg} / \mathrm{d})$ was initiated at $51 \mathrm{st} \mathrm{d}$ of illness. Her abdominal manifestations and proteinuria gradually diminished after starting the PSL. Renal biopsy findings obtained at the 71st d of illness were consistent with those for lupus nephritis (Supplementary Fig. 2).

We conducted a multi-cytokine analysis of the patient's serum and ascites using the Luminex system to clarify the pathophysiology of eosinophilic ascites in the present case. Serum samples in the active phase and during remission, and ascites sample in the active phase were investigated, which revealed elevated interleukin (IL)-5, IL-6, and tumor necrosis factor (TNF)- $\alpha$ levels in ascites and serum IL-6 and

Electronic supplementary material The online version of this article (https://doi.org/10.1007/s12098-019-03092-2) contains supplementary material, which is available to authorized users.

\section{Mamoru Honda}

mamoruhonda8128@gmail.com

1 Department of Pediatrics, National Defense Medical College, 3-2, Namiki, Tokorozawa, Saitama, Japan
TNF- $\alpha$ levels in the active phase. On the other hand, serum IL- 6 and TNF- $\alpha$ levels were decreased in remission (Supplementary Fig. 3).

Previous studies have reported that serum IL-6, IL-10, IL17 , TNF- $\alpha$, and IFN- $\gamma$ levels are elevated in patients with SLE $[2,3]$. In the present case, the IL- 6 and TNF- $\alpha$ levels were elevated in both the serum and ascites samples. Moreover, the IL-5 level was elevated only in ascites, which is characteristic for this case. IL-5, the growth and differentiation factor of eosinophils, is associated with the onset of EGE and eosinophilic infiltration into the gastrointestinal (GI) tract [4]. Our study suggests that eosinophilic ascites in this patient with SLE is the result of IL-5 production of abnormal T lymphocytes in ascites. Atypical cytokine production from abnormal $\mathrm{T}$ lymphocytes in this patient can cause this atypical clinical course similar to that of EGE.

\section{Compliance with Ethical Standards}

Conflict of Interest None.

\section{References}

1. Hochberg MC. Updating the American College of Rheumatology revised criteria for the classification of systemic lupus erythematosus. Arthritis Rheum. 1997;40:1725.

2. Grondal G, Gunnarsson I, Ronnelid J, Rogberg S, Klareskog L, Lundberg I. Cytokine production, serum levels and disease activity in systemic lupus erythematosus. Clin Exp Rheumatol. 2000;18: 565-70.

3. Postal M, Appenzeller S. The role of tumor necrosis factor-alpha $(\mathrm{TNF}-\alpha)$ in the pathogenesis of systemic lupus erythematosus. Cytokine. 2011;56:537-43.

4. Rached AA, El Hajj W. Eosinophilic gastroenteritis: approach to diagnosis and management. World J Gastrointest Pharmacol Ther. 2016;7:513-23.

Publisher's Note Springer Nature remains neutral with regard to jurisdictional claims in published maps and institutional affiliations. 